\title{
Flexible working: being realistic
}

Anonymous

When returning to work after a period of maternity/paternity leave, your attention may inevitably turn to flexible working and the policies that outline this seemingly attractive option. The answer to all your worries and woes about childcare, dropoffs, and pick-ups, while still getting your teaching prepared, your research grant appications written, and all those highimpact papers accepted for publication.

A range of 'voices' may emerge from the mist as you swither about the 'number' of days, which days, condensed hours, compressed hours, after hours, and weekends. "Don't do it - you will still find yourself working full-time" to "Go for it - I wish I had done that - the kids are only wee for such a short time". If discussions with your line manager go well, you may be encouraged to take your time, and have a proper think about it and then submit your application when you are ready.

After much deliberation you submit your flexible working application, and joy of all joys, it is accepted! Just like that - it was easy really after all that worrying, angst, and uncertainty - it has all paid off... Your beautifully balanced and flexible working life has just begun... And then the unforeseen and formidable reality gap emerges right in front of you...

Your letter of confirmation arrives, and there it is in writing: a reduction in hours to a 0.6 contract. Perfect - just what you had asked for! But just a minute, what about the associated reduction to your workload? Ah, then a different set of 'voices' emerge: "Well, everyone is over-worked..." Yes, but you've just taken a huge reduction in your wage to work fewer hours... "Well, no-one is paying me extra to work over my full-time workload, so why should it be any different for you?" Hang on - this wasn't part of the deal (or so you had thought)! All of a sudden the reality of flexibly working a 0.9 workload in a 0.6 contact does not seem quite so attractive. In fact, it is downright stressful! Before you know it you are working just as much as you were before at even more unsociable hours (around pick-ups and drop offs), and being paid just over half of what you had previously earned! The so-called "motherhood penalty" relating to the gender pay gap is well documented, but that is small comfort when you are living with it (Budig \& England, 2001).

\section{Ingredients}

- $\quad$ Flexible working policy.

- Flexible administrator.

- Flexible \& supportive line manager.

- Cognitive flexibility - the human ability to adapt cognitive processing strategies to face new and unexpected conditions.

\section{Method}

1. Speak to as many trusted sources as you can about the flexible working

Flexible working: being realistic EqualBITE 154 
culture at your institution. Do many people have this arrangement and what is their experience of it?

2. If there is not a precedent for this type of working, despite the institution having a flexible working policy, why is this? What are the experiences of those who tried this type of arrangement? Why did it not work? What are the challenges to offering a reduced contract to a member of your staff team?

The University of Edinburgh flexible working policy can be found here: http://www.docs. csg.ed.ac.uk/ HumanResources/ Policies/Flexible Working_Policy.p̄df

3. It can be useful to know the likely tensions and sources of conflict prior to making a new agreement about working hours. For example, who will cover the 'extra' hours that will become available?
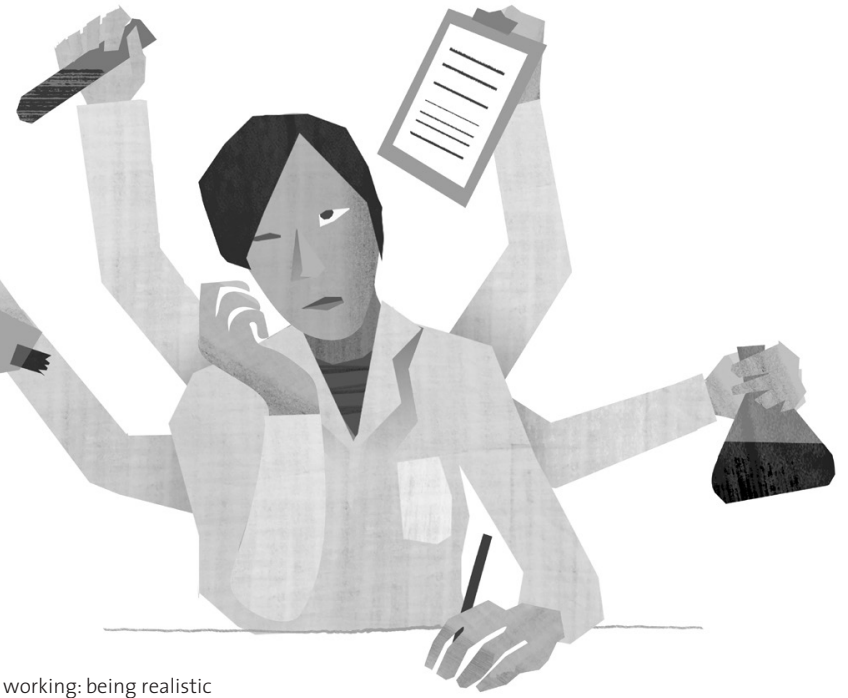


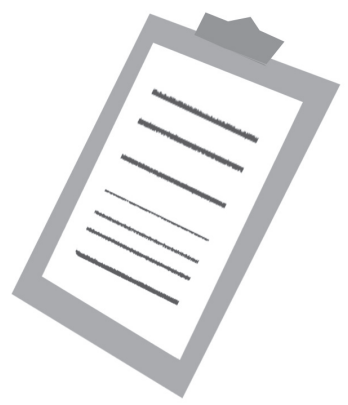

4. Give some thought as to how the new arrangement would look in terms of workload. How would this take shape? What would you or your staff member lose or gain, and how will this be managed?

5. Assess whether you have buy-in from your line manager. Everyone is all too willing to implement a flexible working policy, but will they back and protect a reduced workload or are their hands tied? For example, will there be someone else brought in to pick up the extra work? If not, the chances are you (or they) will still be doing it! Ask about what is not listed (e.g. likelihood of a reduced workload following reduction in hours and practices, policies, and people in place to ensure this).

6. Once you have the warts and all details, and if you still wish to proceed, it can make a lot of sense to push for a temporary reduction in hours for a time-limited period (e.g. until junior goes to school). This way the contract can be adjusted again, more or less, at a later period.

7. It can be extremely difficult in all the chaos and madness of returning to work and flexible working to know what you want, and what might work out best, so a temporary adjustment to your contract leaves your options open for later. 